\title{
Cómo empezar a escribir un Artículo Científico
}

How to star writing a scientific article

Alejandra Vega Barrios ${ }^{a}$, Consuelo Goytortúa Coyoli ${ }^{b}$

\begin{abstract}
:
A scientific article is a specialized work in written format, which aims to describe the theories that support the facts that generated it, the objective of the scientific texts is to explain and shows the researchers results in a clear way, to facilitate their understanding. When an article is started, it starts from a problem that is intended to be studied and that seeks results from quantitative or qualitative methodologies that allow data collection, which can be contrasted to issue results and discussion on chosen theme to found new knowledge.
\end{abstract}

Keywords:

Article, Scientific Texts, methodologies

\section{Resumen:}

Un artículo científico es la redacción de un trabajo especializado en formato escrito, el cual tiene como finalidad describir las teorías que apoyan a los hechos que lo generaron, el objetivo de los textos científicos es explicar y dar a conocer los resultados de las investigaciones que realizan uno o varios autores de forma clara y precisa, para facilitar su comprensión Cuando se inicia un artículo se parte de una problemática que se pretende estudiar y sobre el que buscan resultados a partir de metodologías cuantitativas o cualitativas que permitan la recolección de datos, que pueden ser contrastados para emitir resultados y discusión sobre la temática elegida para abordar nuevos conocimientos.

\section{Palabras Clave:}

Artículo, Textos Científicos, metodologías

\section{Introducción}

El inicio de la escritura de un artículo científico es una forma de explicar sobre lo que se está trabajando, porqué se eligió el tema o la problemática, como se detectó, que se hizo o que metodologías se siguieron para encontrar respuestas a la problemática planteada, cuales fueron los resultados y el análisis de estos resultados que significan para quién se ha determinado que deben conocerse.

La idea fundamental de este artículo es poder dar algunos consejos de como iniciar en esta aventura de escribir y compartir lo que se busca, por qué se busca y que es lo que se encuentra, que servirá a otros para seguir aprendiendo nuevos conocimientos.

\begin{abstract}
Existen algunos procedimientos que podemos iniciar para hacer más sencillo el cómo iniciar con la escritura de un artículo científico como lo es Planificar, dónde una parte importante es tener en consideración en que revista se desea publicar de acuerdo con el interés del investigador, del enfoque de la revista y el tema abordado, las condiciones de publicación y el público destinatario entre otros.
\end{abstract}

Es importante revisar las instrucciones para autores, donde se debe tener presente la cantidad de palabras, cuadros y figuras en cada sección del artículo. [1]

Por otra parte, se debe definir quiénes serán los autores del artículo y en qué orden especifico deberán ser

\footnotetext{
a Autor de Correspondencia, Profesor Investigador del área académica de Mercadotecnia. Universidad Autónoma del Estado de Hidalgo, Instituto de Ciencias Económico-Administrativas, https://orcid.org/0000-0002-2368-7939, Email: vbarrios@uaeh.edu.mx

b Profesor Investigador del área académica de Mercadotecnia. Universidad Autónoma del Estado de Hidalgo, Instituto de Ciencias Económico-Administrativas, https://orcid.org/0000-0003-3956-2046,Email: cgoytortúa@uaeh.edu.mx
} 
colocados, además de elegir el momento para iniciar la escritura.

Al iniciar el desarrollo del artículo, se recomienda hacer una revisión de la bibliografía del tema que se esta trabajando con la intención de conocer otros trabajos con la misma temática, además de apoyarse en un programa de manejo de citas para corregir o entender la citación de acuerdo con las instrucciones de la revista elegida.

Se recomienda realizar un esquema general sobre lo que se desea comunicar con las secciones principales a ser trabajadas, de tal manera que sea sencillo identificar el orden de redacción que debe seguirse, es muy conveniente iniciar de lo general a lo particulary en tiempo presente.

La metodología del artículo comprende la explicación de qué, cuándo, cómo, con qué y para qué con la intención de que otros puedan replicar el trabajo de acuerdo con el contexto que estén viviendo, con la finalidad de obtener los resultados concretos que se comu nicaran, pu diéndose incluir cuadros, figuras, manifestándose en tiempo pasado. . [2]

La Discusión es una sección necesaria donde se interpretan los resultados de otros trabajos con lo realizado para ser contrastados y emitir recomendaciones para otras investigaciones, no olvidando incluir las limitaciones del estudio
Redacte el título y el resumen al final, ya que son las secciones que serán leídas por la mayoría de los lectores y que ayudaran a que se adentren en la lectura o no se tome en cuenta el artículo.

No debemos olvidar la lista de las referencias bibliográficas con el orden secuencial de acuerdo al formato solicitado por la revista, cuide el estilo de redacción y use correctamente el lenguaje científico. Para finalizar debe revisar, revisar y revisar, de presencia con otros investigadores para que puedan identificar errores evidentes, compruebe la coherencia y la claridad de la información, así como los cuadros y figuras, si no se escribe en la legua nativa es necesario solicitar apoyo de correctores de estilo del idioma.

\section{Referencias}

[1] L. Mateu Arrom, J. Huguet, C. Errando, A. Breda, J. Palou. How to write an original article

Actas Urológicas Españolas (English Edition), Volume 42, Issue 9, November 2018, Pages 545-550.

[2] Merino-Trujillo, Alejandra Como escribir documentos científicos (Parte 3). Artículo de revisión. Salud en Tabasco. 2011;17(1-2): undefinedundefined. [fecha de Consulta 2 de septiembre de 2019]. ISSN: $1405-$ $2091 . \quad$ Disponible http://www.redalyc.org/articulo.oa? $\mathrm{id}=487 / 48721182006$

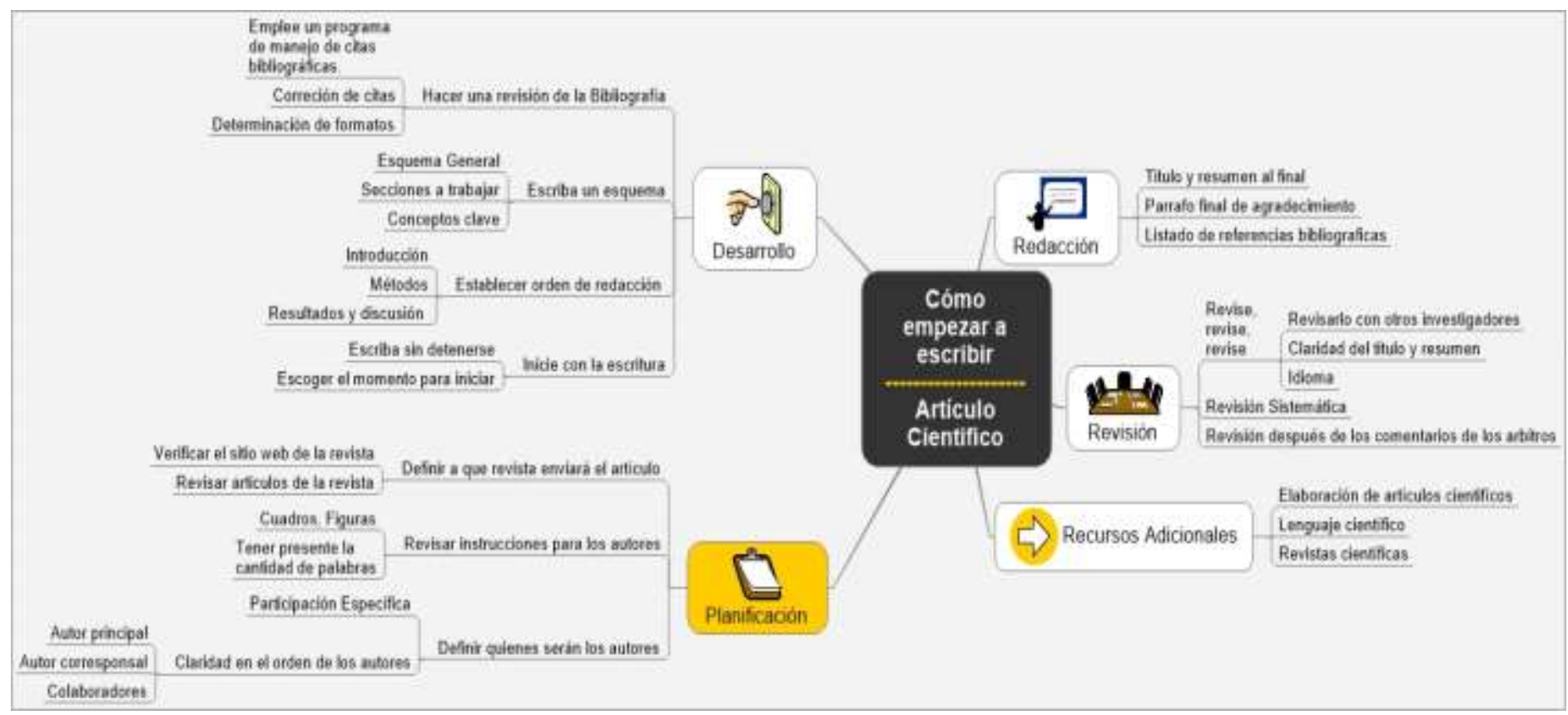

